\title{
A triple product formula for plane partitions derived from biorthogonal polynomials
}

\author{
Shuhei Kamioka1t \\ ${ }^{1}$ Department of Applied Mathematics and Physics, Graduate School of Informatics, Kyoto University, Japan
}

\begin{abstract}
A new triple product formulae for plane partitions with bounded size of parts is derived from a combinatorial interpretation of biorthogonal polynomials in terms of lattice paths. Biorthogonal polynomials which generalize the little $q$-Laguerre polynomials are introduced to derive a new triple product formula which recovers the classical generating function in a triple product by MacMahon and generalizes the trace-type generating functions in double products by Stanley and Gansner.
\end{abstract}

Résumé. Une nouvelle formule pour des partitions planes donnée dans un produit triple est obtenue d'une interprétation combinatoire des polynômes biorthogonaux en termes de chemins sur le réseau carré. Des polynômes biorthogonaux qui généralisent les petits $q$-polynômes de Laguerre sont introduits pour obtenir une nouvelle formule qui généralise la fonction génératrice dans un triple produit établie par McMahon et les fonctions génératrice de traces dans des produits doubles établies par Stanley et Gansner.

Keywords. plane partitions, biorthogonal polynomials, orthogonal polynomials, lattice paths

\section{Introduction}

A plane partition $\pi$ of a nonnegative integer $N$ is a two-dimensional array $\pi=\left(\pi_{i, j}\right)_{i, j=1,2,3, \ldots}$ of nonnegative integers such that $\sum_{i, j=1}^{\infty} \pi_{i, j}=N$ and $\pi_{i, j} \geq \max \left\{\pi_{i+1, j}, \pi_{i, j+1}\right\}$ for every $(i, j) \in \mathbb{Z}_{\geq 1}^{2}$. (Throughout the paper we write $\mathbb{Z}_{\geq k}$ for the set of integers at least $k$.) A plane partition $\pi$ distributes $N$ among its parts $\pi_{i, j}$ so that each row and each column are non-increasing, and gives a two-dimensional analogue of an (integer) partition. MacMahon studied plane partitions in depth and found the following generating function in a triple product [9, Section IX]

$$
\sum_{\pi \in \mathcal{P}(r, c, n)} q^{|\pi|}=\prod_{i=0}^{r-1} \prod_{j=0}^{c-1} \prod_{k=0}^{n-1} \frac{1-q^{i+j+k+2}}{1-q^{i+j+k+1}}
$$

with $|\pi|=\sum_{i, j=1}^{\infty} \pi_{i, j}$ where $\mathcal{P}(r, c, n)$ denotes the set of plane partitions of at most $r$ rows and at most $c$ columns whose parts are bounded above by $n$. Namely $\pi \in \mathcal{P}(r, c, n)$ if and only if $\pi_{r+i, j}=\pi_{i, c+j}=0$ for every $(i, j) \in \mathbb{Z}_{\geq 1}$ and $\pi_{1,1} \leq n$.

\footnotetext{
†Email: kamioka.shuhei.3w@kyoto-u.ac.jp.

1365-8050 @ 2016 Discrete Mathematics and Theoretical Computer Science (DMTCS), Nancy, France
} 
Let $\mathcal{P}(r, c)=\cup_{n=0}^{\infty} \mathcal{P}(r, c, n)$, the set of plane partitions of at most $r$ rows and at most $c$ columns. MacMahon also showed the following generating function in a double product

$$
\sum_{\pi \in \mathcal{P}(r, c)} q^{|\pi|}=\prod_{i=0}^{r-1} \prod_{j=0}^{c-1}\left(1-q^{i+j+1}\right)^{-1}
$$

that is obtained from (1) by $n \rightarrow \infty$. Stanley introduced the $\operatorname{trace} \operatorname{tr}(\pi)=\sum_{i=1}^{\infty} \pi_{i, i}$ of plane partitions and generalized (2) as [11, 12]

$$
\sum_{\pi \in \mathcal{P}(r, c)} q^{|\pi|} a^{\operatorname{tr}(\pi)}=\prod_{i=0}^{r-1} \prod_{j=0}^{c-1}\left(1-a q^{i+j+1}\right)^{-1} .
$$

Gansner later refined (3) as [3, 4]

$$
\sum_{\pi \in \mathcal{P}(r, c)} \prod_{\ell \in \mathbb{Z}} q_{\ell}^{\operatorname{tr}_{\ell}(\pi)}=\prod_{i=0}^{r-1} \prod_{j=0}^{c-1}\left(1-\prod_{\ell=-i}^{j} q_{\ell}\right)^{-1}
$$

with the $\ell$-traces $\operatorname{tr}_{\ell}(\pi)=\sum_{j-i=\ell} \pi_{i, j}$ for $\ell \in \mathbb{Z}$. (Gansner obtained more general results for (reverse) plane partitions of arbitrary shape.)

Gansner's generating function (4) in a double product recovers Stanley's (3) by $q_{\ell}=q$ for all $\ell \in \mathbb{Z}$ except for $q_{0}=a q$, that further recovers MacMahon's (2) by $a=1$. We so have a series of double product formulae (2), (3) and (4) for the set $\mathcal{P}(r, c)$ of plane partitions with unbounded size of parts. Is there an analogous series of triple product formulae for the set $\mathcal{P}(r, c, n)$ of plane partitions with bounded size of parts? We find in this paper such a series of triple product formulae, which involves MacMahon's generating function (1) and generalizations of the trace-type generating functions (3) and (4), with the help of biorthogonal polynomials.

This paper is organized as follows. In Section 2 we explain basics of biorthogonal polynomials and show a combinatorial interpretation of (general) biorthogonal polynomials in terms of lattice paths. A determinant of moments for biorthogonal polynomials will admit a combinatorial expression by nonintersecting lattice paths of Gessel-Viennot [5] type. In Section 3 we introduce specific biorthogonal polynomials, which we call the generalized little $q$-Laguerre polynomials, and examine lattice path combinatorics of the generalized little $q$-Laguerre polynomials based on the general results developed in Section 2. The results in Section 3 are used in Section 4 to derive a triple product formula for plane partitions which generalizes Gansner's generating function (4) (Theorem 9). The triple product formula will reduce to another triple product formula, which will generalize Stanley's generating function (3), and recover MacMahon's generating function (11) by specialization of parameters.

\section{Lattice path combinatorics of biorthogonal polynomials}

Let $\mathbb{K}$ be a field. Let $\mathcal{F}: \mathbb{K}\left[x^{ \pm 1}, y^{ \pm 1}\right] \rightarrow \mathbb{K}$ be a linear functional defined on the space of Laurent polynomials in $x$ and $y$ over $\mathbb{K}$. The linearity of $\mathcal{F}$ means that $\mathcal{F}[a P(x, y)+b Q(x, y)]=a \mathcal{F}[P(x, y)]+$ $b \mathcal{F}[Q(x, y)]$ for any constants $a$ and $b$ and any Laurent polynomials $P(x, y)$ and $Q(x, y)$. The linear functional $\mathcal{F}$ is thus uniquely determined by the moments

$$
f_{i, j}=\mathcal{F}\left[x^{i} y^{j}\right], \quad(i, j) \in \mathbb{Z}^{2} .
$$


We define determinants of moments

$$
\Delta_{n}^{(r, c)}=\operatorname{det}_{0 \leq i, j<n}\left(f_{r+i, c+j}\right), \quad(r, c) \in \mathbb{Z}^{2}, \quad n \in \mathbb{Z}_{\geq 0},
$$

where $\Delta_{0}^{(r, c)}=1$. We assume throughout the paper that the determinant $\Delta_{n}^{(r, c)}$ does not vanish.

Let $(r, c) \in \mathbb{Z}^{2}$ and $n \in \mathbb{Z}_{\geq 0}$. We define a (monic) biorthogonal polynomial $P_{n}^{(r, c)}(x) \in \mathbb{K}[x]$ with the leading term $x^{n}$ by the orthogonality

$$
\mathcal{F}\left[x^{r} y^{c+j} P_{n}^{(r, c)}(x)\right]=h_{n}^{(r, c)} \delta_{j, n}, \quad 0 \leq j \leq n,
$$

where $h_{n}^{(r, c)}$ is some nonvanishing constant, called the normalization constant, and $\delta_{j, n}$ the Kronecker delta. The biorthogonal polynomial uniquely exists for $\mathcal{F}$. (Write down $(7)$ in a linear system of the coefficients of $P_{n}^{(r, c)}(x)$ and solve it.) The monicity and the orthogonality (7) induce the determinant expression of the biorthogonal polynomial

$$
P_{n}^{(r, c)}(x)=\left|\begin{array}{cccccc}
f_{r, c} & \cdots & f_{r, c+j} & \cdots & f_{r, c+n-1} & 1 \\
\vdots & & \vdots & & \vdots & \vdots \\
f_{r+i, c} & \cdots & f_{r+i, c+j} & \cdots & f_{r+i, c+n-1} & x^{i} \\
\vdots & & \vdots & & \vdots & \vdots \\
f_{r+n, c} & \cdots & f_{r+n, c+j} & \cdots & f_{r+n, c+n-1} & x^{n}
\end{array}\right| \times\left(\Delta_{n}^{(r, c)}\right)^{-1}
$$

and hence

$$
h_{n}^{(r, c)}=\frac{\Delta_{n+1}^{(r, c)}}{\Delta_{n}^{(r, c)}} .
$$

We remark that the biorthogonal polynomials $P_{n}^{(r, c)}(x)$ satisfy the biorthogonality relation

$$
\mathcal{F}\left[x^{r} y^{c} P_{m}^{(r, c)}(x) Q_{n}^{(r, c)}(y)\right]=h_{n}^{(r, c)} \delta_{m, n}, \quad m, n \in \mathbb{Z},
$$

with polynomials $Q_{n}^{(r, c)}(y) \in \mathbb{K}[y]$ given by

$$
Q_{n}^{(r, c)}(y)=\left|\begin{array}{ccccc}
f_{r, c} & \cdots & f_{r, c+j} & \cdots & f_{r, c+n} \\
\vdots & & \vdots & & \vdots \\
f_{r+i, c} & \cdots & f_{r+i, c+j} & \cdots & f_{r+i, c+n} \\
\vdots & & \vdots & & \vdots \\
f_{r+n-1, c} & \cdots & f_{r+n-1, c+j} & \cdots & f_{r+n-1, c+n} \\
1 & \cdots & y^{j} & \cdots & y^{n}
\end{array}\right| \times\left(\Delta_{n}^{(r, c)}\right)^{-1}
$$

which may be different from $P_{n}^{(r, c)}(x)$. In the case where $x=y$ the biorthogonal polynomials reduce to ordinary orthogonal polynomials $P_{n}^{(r, c)}(x)=Q_{n}^{(r, c)}(x)$ which are self-orthogonal. See, e.g., [2] for details on orthogonal polynomials. The next proposition shows biorthogonal analogues of the Christoffel and Geronimus transformations for orthogonal polynomials, see, e.g., [13]. 


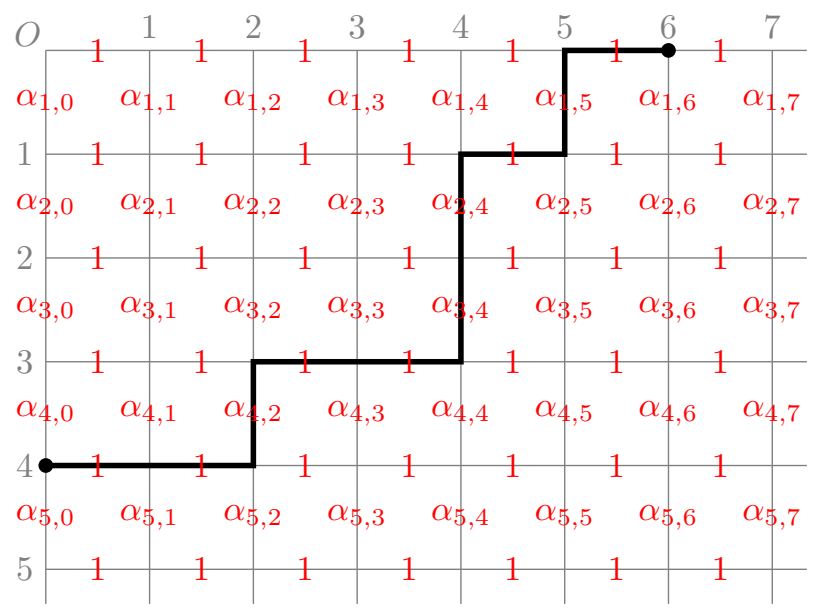

Fig. 1: The square lattice $\mathbb{Z}_{\geq 0}^{2}$ with edges labelled. The thick line is a lattice path going from $(4,0)$ to $(0,6)$.

Proposition 1 (cf. [10]) The biorthogonal polynomials satisfy the adjacent relations

$$
\begin{aligned}
x P_{n}^{(r+1, c)}(x) & =P_{n+1}^{(r, c)}(x)+a_{n}^{(r, c)} P_{n}^{(r, c)}(x), \\
P_{n}^{(r, c)}(x) & =P_{n}^{(r, c+1)}(x)+b_{n}^{(r, c)} P_{n-1}^{(r, c+1)}(x)
\end{aligned}
$$

for $(r, c) \in \mathbb{Z}^{2}$ and $n \in \mathbb{Z}_{\geq 0}$ with $b_{0}^{(r, c)} P_{-1}^{(r, c+1)}(x)=0$ where

$$
a_{n}^{(r, c)}=\frac{h_{n}^{(r+1, c)}}{h_{n}^{(r, c)}}=\frac{\Delta_{n+1}^{(r+1, c)} \Delta_{n}^{(r, c)}}{\Delta_{n}^{(r+1, c)} \Delta_{n+1}^{(r, c)}}, \quad b_{n}^{(r, c)}=\frac{h_{n}^{(r, c)}}{h_{n-1}^{(r, c+1)}}=\frac{\Delta_{n+1}^{(r, c)} \Delta_{n-1}^{(r, c+1)}}{\Delta_{n}^{(r, c)} \Delta_{n}^{(r, c+1)}} .
$$

Proof: Expand $x P_{n}^{(r+1, c)}(x)$ and $P_{n}^{(r, c)}(x)$ in linear combinations of $P_{k}^{(r, c)}(x), 0 \leq k \leq n+1$, and $P_{\ell}^{(r, c+1)}(x), 0 \leq \ell \leq n$, respectively and then equate the coefficients by using the monicity and the orthogonality 7 .

In the rest of this section we show a combinatorial interpretation of biorthogonal polynomials in terms of lattice paths. Let us view a two-dimensional integral lattice in the first quadrant, $\mathbb{Z}_{\geq 0}^{2}$, as a square lattice. We depict the square lattice in matrix-like coordinates where the south and east neighbors of the lattice point $(i, j) \in \mathbb{Z}_{\geq 0}^{2}$ are $(i+1, j)$ and $(i, j+1)$ respectively. For any lattice points $S \in \mathbb{Z}_{\geq 0}^{2}$ and $T \in \mathbb{Z}_{\geq 0}^{2}$ a lattice path $P$ going from $S$ to $T$ is a path on the square lattice which travels between $S$ and $T$ with north steps $(-1,0)$ and east steps $(0,1)$. Figure 1 shows a lattice path going from $(4,0)$ to $(0,6)$. When $S=T$ we conventionally consider the empty lattice path of no steps at $S=T$.

Let $\alpha_{i, j},(i-1, j) \in \mathbb{Z}_{\geq 0}^{2}$, be arbitrary constants. As shown in Figure 1 we label the edges of the square lattice by $\alpha_{i, j}$ or 1 ; the vertical edge between the lattice points $(i, j)$ and $(i-1, j)$ by $\alpha_{i, j}$, and every horizontal edge by 1 . The weight $w(P)$ of a lattice path $P$ is defined to be the product of the labels of all the edges passed by $P$. For example, the lattice path in Figure 1 has the weight $w(P)=\alpha_{4,2} \alpha_{3,4} \alpha_{2,4} \alpha_{1,5}$. The weight of any empty lattice path is assume to be 1 . 
The next theorem gives the base of the combinatorial interpretation of biorthogonal polynomials.

Theorem 2 Assume that

$$
\alpha_{i, j}=a_{j}^{(i-j-1,0)} \quad \text { if } i>j ; \quad=b_{i}^{(0, j-i)} \quad \text { if } i \leq j
$$

where $a_{j}^{(i-j-1,0)}$ and $b_{i}^{(0, j-i)}$ are coefficients of the adjacent relations $\left[12\right.$. For each $(r, c) \in \mathbb{Z}_{\geq 0}^{2}$ then

$$
\frac{f_{r, c}}{f_{0, c}}=\sum_{P} w(P)
$$

where the sum ranges over all the lattice paths $P$ going from $(r, 0)$ to $(0, c)$.

We can confirm Theorem 2 by the adjacent relations (12). For example, let us consider the case where $(r, c)=(2,2)$. We expand the monomial $x^{r}=x^{2}$ in a linear combination of $P_{k}^{(0, c)}(x)=P_{k}^{(0,2)}(x)$ by using (12) as follows. By (12a) with 14,

$$
\begin{aligned}
x^{2}=x^{2} P_{0}^{(2,0)}(x) & =x P_{1}^{(1,0)}(x)+\alpha_{2,0} x P_{0}^{(1,0)}(x) \\
& =P_{2}^{(0,0)}(x)+\left(\alpha_{2,0}+\alpha_{2,1}\right) P_{1}^{(0,0)}(x)+\alpha_{2,0} \alpha_{1,0} P_{0}^{(0,0)}(x) .
\end{aligned}
$$

By (12b) with 14, ,

$$
\begin{aligned}
& =P_{2}^{(0,1)}(x)+\left(\alpha_{2,0}+\alpha_{2,1}+\alpha_{2,2}\right) P_{1}^{(0,1)}(x)+\left(\alpha_{2,0} \alpha_{1,1}+\alpha_{2,1} \alpha_{1,1}+\alpha_{2,0} \alpha_{1,0}\right) P_{0}^{(0,1)}(x) \\
& =Q(x)+\left(\alpha_{2,0} \alpha_{1,2}+\alpha_{2,1} \alpha_{1,2}+\alpha_{2,2} \alpha_{1,2}+\alpha_{2,0} \alpha_{1,1}+\alpha_{2,1} \alpha_{1,1}+\alpha_{2,0} \alpha_{1,0}\right) P_{0}^{(0,2)}(x)
\end{aligned}
$$

where $Q(x)$ is a linear combination of $P_{1}^{(0,2)}(x)$ and $P_{2}^{(0,2)}(x)$. In the last equation the coefficient of $P_{0}^{(0,2)}(x)$ is equal to the right-hand sum of $[15]$. Thus

$$
x^{2}=Q(x)+P_{0}^{(0,2)}(x) \sum_{P} w(P) .
$$

For this expansion of $x^{2}$ we multiply $y^{c}=y^{2}$ to the both sides and apply the linear functional $\mathcal{F}$. From the orthogonality (7) we then obtain

$$
f_{2,2}=\mathcal{F}\left[y^{2} Q(x)\right]+\mathcal{F}\left[y^{2} P_{0}^{(0,2)}(x)\right] \sum_{P} w(P)=h_{0}^{(0,2)} \sum_{P} w(P)
$$

that gives [15] since $h_{0}^{(0,2)}=f_{0,2}$. See [6] for the complete proof of Theorem 2 .

Theorem 2 provides a combinatorial interpretation of moments of biorthogonal polynomials in terms of lattice paths. In view of Gessel-Viennot's method [5], [1, Chapter 31], that naturally leads to a combinatorial interpretation of determinants of moments in terms of non-intersecting lattice paths. For $(r, c, n) \in \mathbb{Z}_{\geq 0}^{3}$ we define $\mathcal{L P}(r, c, n)$ to be the set of $n$-tuples $\left(P_{0}, \ldots, P_{n-1}\right)$ of lattice paths such that (i) $P_{k}$ goes from $(r+k, 0)$ to $(0, c+k)$; (ii) $P_{0}, \ldots, P_{n-1}$ are non-intersecting, namely $P_{j} \cap P_{k}=\emptyset$ if $j \neq k$. Figure 2 shows an example of such an $n$-tuple $\left(P_{0}, \ldots, P_{n-1}\right) \in \mathcal{L} \mathcal{P}(r, c, n)$ when $(r, c, n)=(4,5,3)$.

Corollary 3 For each $(r, c, n) \in \mathbb{Z}_{\geq 0}^{3}$,

$$
\frac{\Delta_{n}^{(r, c)}}{\prod_{k=0}^{n-1} f_{0, c+k}}=\sum_{\left(P_{0}, \ldots, P_{n-1}\right) \in \mathcal{L} \mathcal{P}(r, c, n)} \prod_{k=0}^{n-1} w\left(P_{k}\right) .
$$




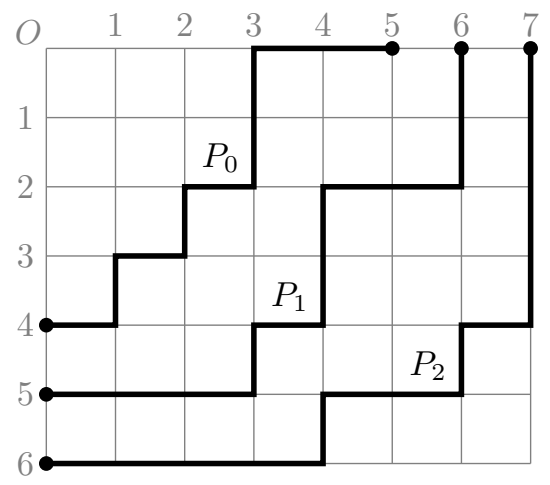

Fig. 2: An $n$-tuple $\left(P_{0}, \ldots, P_{n-1}\right) \in \mathcal{L} \mathcal{P}(r, c, n)$ of non-intersecting lattice paths on the square lattice $\mathbb{Z}_{\geq 0}^{2}$ where $(r, c, n)=(4,5,3)$.

\section{Generalized little $q$-Laguerre polynomials}

In this section we apply the combinatorial interpretation of (general) biorthogonal polynomials in the previous section to a specific instance of biorthogonal polynomials which we call the generalized little $q$-Laguerre polynomials. In what follows we adopt the following notations. For any sequence $x_{\ell}, \ell \in \mathbb{Z}$,

$$
[x]_{m}^{n}=\prod_{k=m}^{n} x_{k} \quad \text { if } m \leq n ; \quad=\prod_{k=n+1}^{m-1} x_{k}^{-1} \quad \text { if } m>n
$$

where $[x]_{n+1}^{n}=1$.

Let $a, p_{\ell}$ and $q_{\ell}, \ell \in \mathbb{Z}$, be indeterminates. We define the generalized little $q$-Laguerre polynomial of degree $n$ by

$$
\mathcal{L}_{n}\left(x ; a ; p_{1}, \ldots, p_{n-1} ; q_{1}, \ldots, q_{n-1}\right)=\sum_{i=0}^{n} x^{i}\left(\prod_{k=i}^{n-1}[p]_{1}^{k}\right) \sum_{i \geq \nu_{i} \geq \cdots \geq \nu_{n-1} \geq 0} \prod_{k=i}^{n-1}\left(a[q]_{1}^{k-\nu_{k}}-\frac{1}{[p]_{1}^{\nu_{k}}}\right) .
$$

The name comes from the (monic) little q-Laguerre polynomial (cf. [7, §14.20])

$$
(-1)^{n} q^{\frac{n(n-1)}{2}}(a ; q)_{n} \times{ }_{2} \phi_{1}\left(\begin{array}{c}
q^{-n}, 0 \\
a
\end{array} ; q, x q\right)
$$

that is obtained from (21) with specialized parameters $p_{\ell}=q_{\ell}=q$ for all $\ell \in \mathbb{Z}$. (For the standard notations for $q$-analysis, such as $(a ; q)_{n}$ and ${ }_{2} \phi_{1}$, see, e.g., [7] Ch. 1].) Let

$$
\mathcal{L}_{n}^{(r, c)}(x)=\mathcal{L}_{n}\left(x ; a[p]_{1}^{r}[q]_{1}^{c} ; p_{r+1}, \ldots, p_{r+n-1} ; q_{c+1}, \ldots, q_{c+n-1}\right), \quad(r, c) \in \mathbb{Z}^{2}, \quad n \in \mathbb{Z}_{\geq 0} .
$$

Theorem 4 Let $\mathbb{K}=\mathbb{Q}\left(a, p_{0}, p_{ \pm 1}, p_{ \pm 2}, \ldots, q_{0}, q_{ \pm 1}, q_{ \pm 2}, \ldots\right)$. The generalized little $q$-Laguerre polynomials satisfy the orthogonality (7) with $P_{n}^{(r, c)}(x)=\mathcal{L}_{n}^{(r, c)}(x)$ and the normalization constants

$$
h_{n}^{(r, c)}=f_{r, c+n} \times a^{n} \prod_{k=0}^{n-1}[p]_{1}^{r+k}\left([q]_{1}^{c+k}-[q]_{1}^{c+n}\right)
$$


where the linear functional $\mathcal{F}$ has the moments $f_{i, j}=\mathcal{F}\left[x^{i} y^{j}\right]$ given by

$$
f_{i, j}=\prod_{k=0}^{i-1}\left(1-a[p]_{1}^{k}[q]_{1}^{j}\right) \quad \text { if } i \geq 0 ; \quad=\prod_{k=i}^{-1}\left(1-a[p]_{1}^{k}[q]_{1}^{j}\right)^{-1} \quad \text { if } i<0
$$

where $f_{0, j}=1$.

We can prove the orthogonality by means of a generalization of the $q$-Chu-Vandermonde identity for ${ }_{2} \phi_{1}$, see [6] for details. Proposition 1 and Theorem 4 immediately yield the following.

Corollary 5 The generalized little q-Laguerre polynomials satisfy the adjacent relations (12) with $P_{n}^{(r, c)}(x)=$ $\mathcal{L}_{n}^{(r, c)}(x)$ and the coefficients

$$
a_{n}^{(r, c)}=[p]_{r+1}^{r+n}\left(1-a[p]_{1}^{r}[q]_{1}^{c+n}\right), \quad b_{n}^{(r, c)}=a[p]_{1}^{r+n-1}[q]_{1}^{c}\left(1-[q]_{c+1}^{c+n}\right)
$$

The general results in Section 2 gives us the following combinatorial interpretation of the the generalized little $q$-Laguerre polynomials. See [6] for the proofs of Lemma 6 and Theorems 7 and 8 mentioned below.

Let $P$ be a lattice path going from $(r, 0)$ to $(0, c)$. We can see the region bordered by $P$ and the two axes as a Young diagram of an (integer) partition for which we write $\lambda(P)=\left(\lambda_{i}(P)\right)_{i=1,2, \ldots}$ where $\lambda_{i}(P)$ denotes the $i$-th part of the partition $\lambda(P)$. For example, the lattice path in Figure 1 has $\lambda(P)=(5,4,4,2)$. For any Young diagram $\lambda$ we define $\mathrm{D}_{\ell}(\lambda), \ell \in \mathbb{Z}$, to be the number of boxes on the $\ell$-th diagonal of $\lambda$ where a box at $(i, j)$ is on the $\ell$-th diagonal if and only if $j-i=\ell$. For example, $\left(\mathrm{D}_{\ell}(\lambda)\right)_{-3 \leq \ell \leq 5}=$ $(1,2,2,3,3,2,1,1,0)$ for $\lambda=(5,4,4,2)$.

The labels

$$
\alpha_{i, j}=[p]_{i-j}^{i-1}\left(1-a[p]_{1}^{i-j-1}[q]_{1}^{j}\right) \quad \text { if } i>j ; \quad=a[p]_{1}^{i-1}[q]_{1}^{j-i}\left(1-[q]_{j-i+1}^{j}\right) \quad \text { if } i \leq j
$$

from the coefficients 26 of adjacent relations give rise to the following weight.

Lemma 6 Assume (27). Let $P$ be a lattice path going from $(r, 0)$ to $(0, c)$. Then

$$
\begin{aligned}
& w(P)=a^{\mathrm{D}_{0}(\lambda(P))}\left(\prod_{i=1}^{r-1} p_{i}^{\mathrm{D}_{-i}(\lambda(P))}\right)\left(\prod_{j=1}^{c-1} q_{j}^{\mathrm{D}_{j}(\lambda(P))}\right) \omega_{r}^{\prime}(P) \quad \text { with } \\
& \omega_{r}^{\prime}(P)=\left\{\prod_{i=1}^{d}\left(1-[q]_{\lambda_{i}(P)-i+1}^{\lambda_{i}(P)}\right)\right\}\left\{\prod_{i=d+1}^{r}\left(1-a[p]_{1}^{i-\lambda_{i}(P)-1}[q]_{1}^{\lambda_{i}(P)}\right)\right\}
\end{aligned}
$$

where $d=\mathrm{D}_{0}(\lambda(P))$ in $28 \mathrm{~b}$ ).

Theorem 2 when applied to the generalized little $q$-Laguerre polynomials implies the following.

Theorem 7 For each $(r, c) \in \mathbb{Z}_{\geq 0}^{2}$ the moment $f_{r, c}$ given by 25] admits the combinatorial expression

$$
f_{r, c}=\sum_{P} a^{\mathrm{D}_{0}(\lambda(P))}\left(\prod_{i=1}^{r-1} p_{i}^{\mathrm{D}_{-i}(\lambda(P))}\right)\left(\prod_{j=1}^{c-1} q_{j}^{\mathrm{D}_{j}(\lambda(P))}\right) \omega_{r}^{\prime}(P)
$$

where the sum ranges over all the lattice paths going from $(r, 0)$ to $(0, c)$. 


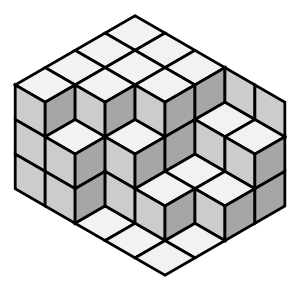

Fig. 3: The 3D Young diagram of the plane partition 31.

The analogue of Corollary 3 for the generalized little $q$-Laguerre polynomials is the following.

Theorem 8 For each $(r, c, n) \in \mathbb{Z}_{\geq 0}^{3}$ the determinant $\Delta_{n}^{(r, c)}=\operatorname{det}_{0 \leq i, j<n}\left(f_{r+i, c+j}\right)$ of the moments $f_{i, j}$ given by 25] satisfies

$$
\begin{aligned}
\Delta_{n}^{(r, c)}\left[\left\{\prod_{k=0}^{n-1}\left(p_{r+k} q_{c+k}\right)^{\frac{(n-k)(n-k-1)}{2}}\right\}\left\{\prod_{1 \leq i \leq k<n}\left(1-[q]_{c+k-i+1}^{c+k}\right)\right\}\right]^{-1} \\
=\sum_{\left(P_{0}, \ldots, P_{n-1}\right) \in \mathcal{L} \mathcal{P}(r, c, n)} a^{\sum_{k=0}^{n-1} \mathrm{D}_{0}\left(\lambda\left(P_{k}\right)\right)}\left(\prod_{i=1}^{r-1} p_{i}^{\sum_{k=0}^{n-1} \mathrm{D}_{-i}\left(\lambda\left(P_{k}\right)\right)}\right)\left(\prod_{j=1}^{c-1} q_{j}^{\sum_{k=0}^{n-1} \mathrm{D}_{j}\left(\lambda\left(P_{k}\right)\right)}\right) \\
\times \omega_{r, n}^{\prime}\left(P_{0}, \ldots, P_{n-1}\right)
\end{aligned}
$$

with

$$
\omega_{r, n}^{\prime}\left(P_{0}, \ldots, P_{n-1}\right)=\prod_{k=0}^{n-1}\left\{\prod_{i=k+1}^{d_{k}}\left(1-[q]_{\lambda_{i}\left(P_{k}\right)-i+1}^{\lambda_{i}\left(P_{k}\right)}\right\}\left\{\prod_{i=d_{k}+1}^{r+k}\left(1-a[p]_{1}^{i-\lambda_{i}\left(P_{k}\right)-1}[q]_{1}^{\lambda_{i}\left(P_{k}\right)}\right)\right\}\right.
$$

where $d_{k}=\mathrm{D}_{0}\left(\lambda\left(P_{k}\right)\right)$.

\section{Triple product formulae for plane partitions}

It is customary to depict a plane partition $\pi=\left(\pi_{i, j}\right)_{i, j=1,2,3, \ldots}$ in a three-dimensional (3D) Young diagram in which $\pi_{i, j}$ (unit) cubes are stacked over the positions $(i, j) \in \mathbb{Z}_{\geq 1}^{2}$. For example, the plane partition

$$
\left(\begin{array}{lllll}
3 & 3 & 3 & 2 & 2 \\
3 & 3 & 3 & 1 & 1 \\
3 & 3 & 2 & 1 & 0 \\
3 & 2 & 0 & 0 & 0
\end{array}\right)
$$

is depicted as the 3D Young diagram shown in Figure 3. For each $k \in \mathbb{Z}_{\geq 1}$ we define a partition $\lambda_{k}(\pi)$ so that the Young diagram of $\lambda_{k}(\pi)$ is equal to the cross-section at level $k$ of the 3D Young diagram of $\pi$. For example, the plane partition [31, or the 3D Young diagram in Figure 3 , gives rise to the partitions

$$
\lambda_{1}(\pi)=(5,5,4,2), \quad \lambda_{2}(\pi)=(5,3,3,2), \quad \lambda_{3}(\pi)=(3,3,2,1)
$$



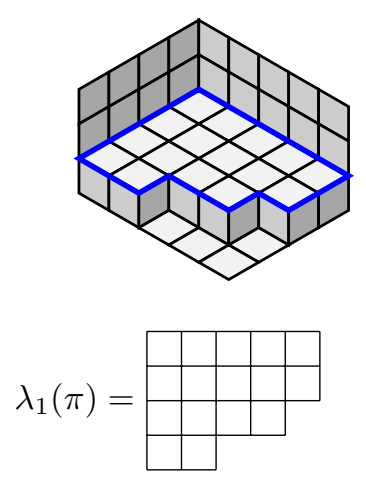
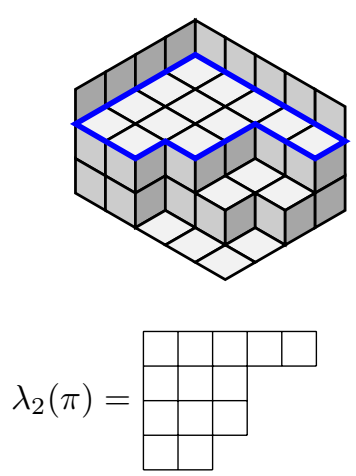
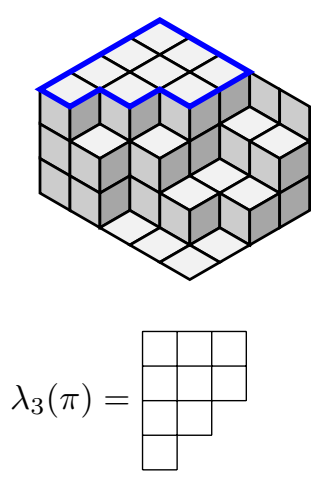

Fig. 4: The cross-sections $\lambda_{k}(\pi)$ at level $k=1,2,3$ of the 3D Young diagram $\pi$ in Figure 3

and $\lambda_{k}(\pi)=\emptyset=(0,0,0, \ldots)$ for $k \geq 4$, see Figure 4. We will write $\lambda_{k, i}(\pi)$ for the $i$-th part of the partition $\lambda_{k}(\pi)$.

There exists a classical bijection between $\mathcal{P}(r, c, n)$ and $\mathcal{L} \mathcal{P}(r, c, n)$ which connects plane partitions with non-intersecting lattice paths. For completeness we here describe the bijection. From a plane partition $\pi \in \mathcal{P}(r, c, n)$ an $n$-tuple $\left(P_{0}, \ldots, P_{n-1}\right) \in \mathcal{L P}(r, c, n)$ of non-intersecting lattice paths corresponding to $\pi$ is constructed as follows.

(i) On the square lattice $\mathbb{Z}_{\geq 0}^{2}$ draw lattice paths $P_{0}^{\prime}, \ldots, P_{n-1}^{\prime}$ going from $(r, 0)$ to $(0, c)$ so that $\lambda\left(P_{k}^{\prime}\right)=$ $\lambda_{n-k}(\pi)$.

(ii) For each $0 \leq k<n$ translate the lattice path $P_{k}^{\prime}$ by $(k, k)$ (so that $P_{k}^{\prime}$ goes from $(r+k, k)$ to $(k, c+k))$. We write $P_{k}^{\prime \prime}$ for the obtained lattice path.

(iii) For each $0 \leq k<n$ add $k$ consecutive east and north steps to the initial and terminal points of $P_{k}^{\prime \prime}$ respectively (so that $P_{k}^{\prime \prime}$ goes from $(r+k, 0)$ to $(0, c+k)$ ). The obtained lattice path is $P_{k}$.

See Figure 5 that demonstrates the construction.

The constructive bijection can be formulated by

$$
\lambda\left(P_{k}\right)=(\underbrace{c, \ldots, c}_{k \text { times }}, \lambda_{n-k, 1}(\pi), \ldots, \lambda_{n-k, r}(\pi))+\left(k^{r+k}\right), \quad 0 \leq k<n .
$$

We hence have

$$
\begin{gathered}
\mathrm{D}_{\ell}\left(\lambda\left(P_{k}\right)\right)=\mathrm{D}_{\ell}\left(\lambda_{n-k}(\pi)\right)+k, \quad \\
\lambda_{i}\left(P_{k}\right)=\lambda_{n-k, i-k}(\pi)+k, \quad \ell \in \mathbb{Z}, \quad 0 \leq k<n, \quad i>k .
\end{gathered}
$$

By means of the bijection, that admits [34, we can translate Theorem 8 for non-intersecting lattice paths into the following theorem for plane partitions, that is the main theorem of this paper. 


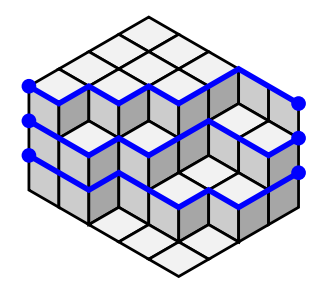

(i)

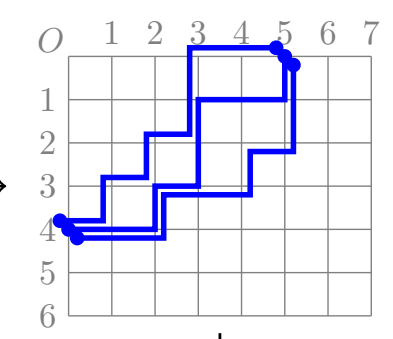

(ii)
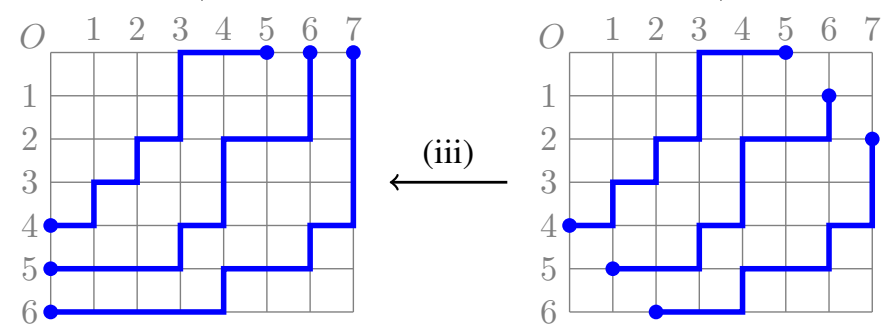

Fig. 5: The bijection between $\mathcal{P}(r, c, n)$ and $\mathcal{L} \mathcal{P}(r, c, n)$ with $(r, c, n)=(4,5,3)$.

Theorem 9 Let $(r, c, n) \in \mathbb{Z}_{\geq 0}^{3}$. Then

$$
\sum_{\pi \in \mathcal{P}(r, c, n)} a^{\operatorname{tr}_{0}(\pi)}\left(\prod_{i=1}^{r-1} p_{i}^{\operatorname{tr}_{-i}(\pi)}\right)\left(\prod_{j=1}^{c-1} q_{j}^{\operatorname{tr}_{j}(\pi)}\right) \omega_{r, n}(\pi)=\prod_{i=0}^{r-1} \prod_{j=0}^{c-1} \prod_{k=0}^{n-1} \frac{1-a[p]_{1}^{i}[q]_{1}^{j+k+1}}{1-a[p]_{1}^{i}[q]_{1}^{j+k}}
$$

with

$$
\begin{array}{r}
\omega_{r, n}(\pi)=\prod_{k=1}^{\pi_{1,1}}\left\{\prod_{i=1}^{d_{k}}\left(1-[q]_{\lambda_{k, i}(\pi)-i+1}^{\lambda_{k, i}(\pi)+n-k}\right)\right\}\left\{\prod_{i=d_{k}+1}^{r}\left(1-a[p]_{1}^{i-\lambda_{k, i}(\pi)-1}[q]_{1}^{n-k+\lambda_{k, i}(\pi)}\right)\right\} \\
\times\left\{\prod_{i=1}^{r}\left(1-a[p]_{1}^{i-1}[q]_{1}^{n-k}\right)\right\}^{-1}
\end{array}
$$

where $\pi_{1,1}$ denotes the $(1,1)$-part of a plane partition $\pi$, and $d_{k}=\mathrm{D}_{0}\left(\lambda_{k}(\pi)\right)$.

Proof: By use of the bijection between $\mathcal{P}(r, c, n)$ and $\mathcal{L P}(r, c, n)$ and 34 we can equivalently translate the formula 30 in Theorem 8 into

$$
\sum_{\pi \in \mathcal{P}(r, c, n)} a^{\operatorname{tr}_{0}(\pi)}\left(\prod_{i=1}^{r-1} p_{i}^{\operatorname{tr}_{-i}(\pi)}\right)\left(\prod_{j=1}^{c-1} q_{j}^{\operatorname{tr}_{j}(\pi)}\right) \omega_{r, n}(\pi)=\frac{\Delta_{n}^{(r, c)}}{\kappa_{n}^{(r, c)}}\left\{\prod_{i=0}^{r-1} \prod_{k=0}^{n-1}\left(1-a[p]_{1}^{i}[q]_{1}^{k}\right)\right\}^{-1}
$$


where

$$
\begin{aligned}
\kappa_{n}^{(r, c)}=\left\{a\left(\prod_{i=1}^{r-1} p_{i}\right)\left(\prod_{j=1}^{c-1} q_{j}\right)\right\}^{\frac{n(n-1)}{2}} & \left\{\prod_{k=0}^{n-1}\left(p_{r+k} q_{c+k}\right)^{\frac{(n-k)(n-k-1)}{2}}\right\} \\
& \times\left\{\prod_{1 \leq i \leq k<n}\left(1-[q]_{c+k-i+1}^{c+k}\right)\right\} .
\end{aligned}
$$

The proof thus amounts to the evaluation of the determinant $\Delta_{n}^{(r, c)}$ of moments for the generalized little $q$-Laguerre polynomials discussed in Section 3 . One possible way to evaluate the determinant is to utilize one of the product formulae for determinants by Krattenthaler [8]. We here show an alternative way based on biorthogonal polynomials. From (9) we have

$$
\Delta_{n}^{(r, c)}=\prod_{k=0}^{n-1} h_{k}^{(r, c)}
$$

for (general) biorthogonal polynomials. Substituting the normalization constants 24) for the generalized little $q$-Laguerre polynomials we readily know

$$
\Delta_{n}^{(r, c)}=\kappa_{n}^{(r, c)} \prod_{i=0}^{r-1} \prod_{k=0}^{n-1}\left(1-a[p]_{1}^{i}[q]_{1}^{c+k}\right) .
$$

Substituting (38) for 36a) we soon get the triple product formula 35a).

The triple product formula (35) for plane partitions reduces to

$$
\begin{aligned}
\sum_{\pi \in \mathcal{P}(r, c, n)} q^{|\pi|} a^{\operatorname{tr}(\pi)} \omega_{n}(\pi) & =\prod_{i=0}^{r-1} \prod_{j=0}^{c-1} \prod_{k=0}^{n-1} \frac{1-a q^{i+j+k+2}}{1-a q^{i+j+k+1}} \quad \text { with } \\
\omega_{n}(\pi) & =\prod_{k=1}^{\pi_{1,1}} \frac{\left(q^{n-k+1} ; q\right)_{\mathrm{D}_{0}\left(\lambda_{k}(\pi)\right)}}{\left(a q^{n-k+1} ; q\right)_{\mathrm{D}_{0}\left(\lambda_{k}(\pi)\right)}}
\end{aligned}
$$

with the specialized parameters $a \leftarrow a q$ and $p_{\ell}=q_{\ell}=q$ for all $\ell$. Furthermore (39) reduces to MacMahon's triple product formulae (1) with $a=1$ since $\left.\omega_{n}(\pi)\right|_{a=1}=1$. We have thus obtained a series of the triple product formulae (1), (39) and (35) for the set $\mathcal{P}(r, c, n)$ of plane partitions with bounded size of parts.

This series of triple product formulae is totally analogous to the series of the generating functions (2), (3) and (4) in double products for the set $\mathcal{P}(r, c)$ of plane partitions with unbounded size of parts. Indeed the flow of reductions from (35) via (39) to (1) for triple product formulae is performed by the same specializations of parameters as the flow of reductions from (4) via (3) to (2) for double product ones, where $q_{0}=a$ and $q_{-\ell}=p_{\ell}$ for all $\ell \in \mathbb{Z}_{>1}$ in (4). Moreover, as MacMahon's generating function (1) in a triple product recovers his generating function (2) in a double product by $n \rightarrow \infty$, the triple product formulae (35) and (39) respectively recover Gansner's and Stanley's generating functions (4) and (3) in 
double products by $n \rightarrow \infty$. In fact $\omega_{r, n}(\pi)$ in (35) and $\omega_{n}(\pi)$ in (39) both tends to 1 , as formal power series in $q_{1}, q_{2}, \ldots$ and in $q$ respectively, as $n \rightarrow \infty$. The series of the triple product formulae (1), (39) and (35) therefore generalizes the series of the generating functions (2), (3) and (4) in double products by an additional parameter $n$ for the upper bound of parts.

\section{Acknowledgements}

The author would like to express his deep gratitude to the anonymous reviewers for their valuable comments and suggestions to improve the quality of the manuscript. The author is also much grateful to Mr. Kazuhiro Morii for his assistance in preparing the manuscript.

\section{References}

[1] M. Aigner and G. M. Ziegler. Proofs from The Book. Springer-Verlag, Berlin, fifth edition, 2014.

[2] T. S. Chihara. An Introduction to Orthogonal Polynomials. Mathematics and its Applications, Vol. 13. Gordon and Breach Science Publishers, New York-London-Paris, 1978.

[3] E. R. Gansner. The enumeration of plane partitions via the Burge correspondence. Illinois J. Math., 25:533-554, 1981.

[4] E. R. Gansner. The Hillman-Grassl correspondence and the enumeration of reverse plane partitions. J. Combin. Theory Ser. A, 30:71-89, 1981.

[5] I. Gessel and G. Viennot. Binomial determinants, paths, and hook length formulae. Adv. in Math., 58:300-321, 1985.

[6] S. Kamioka. Plane partitions with bounded size of parts and biorthogonal polynomials. arXiv:1508.01674.

[7] R. Koekoek, P. A. Lesky, and R. F. Swarttouw. Hypergeometric Orthogonal Polynomials and Their q-Analogues. Springer Monographs in Mathematics. Springer-Verlag, Berlin, 2010.

[8] C. Krattenthaler. Advanced determinant calculus. Sém. Lothar. Combin., 42:Art. B42q (electronic), 1999.

[9] P. A. MacMahon. Combinatory Analysis, Volume 2. Cambridge University Press, Cambridge, 1916.

[10] K. Maeda, H. Miki, and S. Tsujimoto. From orthogonal polynomials to integrable systems. Trans. Jpn. Soc. Ind. Appl. Math., 23:341-380, 2013.

[11] R. P. Stanley. Theory and application of plane partitions, I, II. Studies in Appl. Math., 50:167-188, 259-279, 1971.

[12] R. P. Stanley. The conjugate trace and trace of a plane partition. J. Combinatorial Theory Ser. A, 14: 53-65, 1973.

[13] A. Zhedanov. Rational spectral transformations and orthogonal polynomials. J. Comput. Appl. Math., 85:67-86, 1997. 